\title{
Spacetime Discreteness: Shedding Light on Two of the Simplest Observations in Physics
}

\author{
Ahmed Isam \\ Independent Researcher, Khartoum, Sudan \\ Email: aif.alkab@gmail.com
}

How to cite this paper: Isam, A. (2018) Spacetime Discreteness: Shedding Light on Two of the Simplest Observations in Physics. Journal of Modern Physics, 9, 14151431.

https://doi.org/10.4236/jmp.2018.97086

Received: January 20, 2018

Accepted: June 16, 2018

Published: June 19, 2018

Copyright $\odot 2018$ by author and Scientific Research Publishing Inc. This work is licensed under the Creative Commons Attribution International License (CC BY 4.0).

http://creativecommons.org/licenses/by/4.0/

(c) (i) Open Access

\begin{abstract}
Loop quantum gravity is considered to be one of the two major candidates for a theory of quantum gravity. The most appealing aspect about this theory is it predicts that spacetime is not continuous; both space and time have a discrete nature. Simply, space is not infinitely divisible, but it has a granular structure, and time does not flow continuously like a smooth river. This paper demonstrates a review for two missed (unnoted) observations that support the discreteness of the spacetime. The content of this paper does not validate the specific model of quantized geometry of the spacetime which is predicted by the theory itself. Instead, it proves that time does not flow continuously. But it flows in certain, discrete steps, like a ticking of a clock, due to a simple observation which is absence of any possible value of time that can exist between the present and the future. Regarding space, it validates the spatial discreteness, and the existence of spatial granules (space quanta) due to a simple observation which is the existence of the origin position in a coordinates system. All of this is achieved by reviewing the concept of discreteness itself, and applied directly to the observations.
\end{abstract}

\section{Keywords}

Quantum Gravity, Loop Quantum Gravity, Discrete Time, Discrete Space, Emptiness, Planck Length, Planck Time, Infinities Problem

\section{Introduction}

The problem of quantum gravity represents one of the biggest problems in physics today. Mainly, the problem does not arise from the lack of working theories in this field, but it arises-till now-from absence of any experiment or observation that can validate any theory in this field. The problem comes from the fact that the theories of quantum gravity work on a very small length scale 
( $\sqrt{\hbar G / c^{3}} \approx 10^{-35} \mathrm{~m}$ ), that is beyond our current experimental reach. Therefore, no evidence has been obtained to validate any theory in this field. Loop quantum gravity (LQG) is considered to be one of the two major candidates for a theory of quantum gravity [1] [2] [3], and String theory is the other candidate. Mathematically, the framework of String theory [4] requires the spacetime to have extra dimensions beside the four dimensions that we currently observe (length, width, height, and time). Also, it demands the existence of a specific type of symmetry for the spacetime, which is called supersymmetry. This symmetry implies that each elementary particle should have another particle as a partner (super-partner). A fermion should has a partner boson and vice versa. But the problem with all this is that none of the above until now has been validated by any experiment or observation, not even after the launch of the Large Hadron Collider (LHC) in Europe. Technically, the experts require more time and effort on the experimental research in order to reach their final conclusions. But for now, I think this should raise many questions.

On the other hand, LQG in its current theoretical framework does not require the unobservable extra dimensions or the undiscovered supersymmetry. Also, its experimental future is much more promising. A few years ago, a group of scientists from America and France have proposed a new approach to test LQG [5]. Their proposal depends on detecting the radiation that is emitted from the black holes. Historically, the idea that a black hole can radiate was introduced in 1974 by the British physicist Stephen Hawking [6]. According to his theoretical model, the radiation does not come directly from the black hole itself. But it comes from the quantum effects-due to the uncertainty principle-that exists near the event horizon. Emitting this radiation causes a black hole to loss energy (mass), and from here it is also called black hole evaporation. In their proposal [5], by including LQG in the picture, the process of radiation should reveal footprints that are distinct from the usual outcome which is expected from the process of Hawking radiation. But a major challenge for this test is that the process of black holes radiation is just a hypothesis. Until now, this radiation has not ever been detected. Therefore, in order to verify LQG by this approach, first, we need to prove that this radiation does really exist. Then, we shall look for the characteristic footprints in the radiation process that distinguishes LQG. I hope this can be achieved in the near future.

Theoretically, the most appealing aspect about LQG is that it predicts that spacetime is a discrete entity; space is not infinitely divisible, but it has a granular structure and time flows in a discrete pattern like a ticking of a clock. LQG draws an accurate geometry for the spacetime at the very small scale (Planck length). The theory has its early beginning in the mid-twentieth century. It has been developed by a number of physicists including; Carlo Rovelli, Abhay Ashtekar, and Lee Smolin. It was built in order to merge Einstein's idea of gravity with the quantum theory. In Einstein's notion of gravity [7] [8], the gravitational field is just a curvature in the space-time itself. Therefore, the space-time (our 
background) is an active field, and not just a passive entity where other interactions happen within. In the quantum theory, the traditional description of a field (like Electromagnetic field) is built to rely on a passive background, and here a spontaneous problem emerges. Because Einstein's theory of general relativity tells us that the universe is built by fields on fields interactions, and not by fields interactions on a passive background (fixed, inactive spacetime). The gravitational field does not require a background to rely on, it is the background itself. From here, a need for a new concept becomes prominent in order to merge gravity within the quantum realm. LQG is concerned with this. It describes a new quantum field for gravity that can interact with the other forces, with no fixed background to rely on.

It describes space as network of intersecting loops Figure 1(a). These loops are not located within space, but they are the space. They are excitations of the gravitational field at a very small scale (Planck length). These loops interact with our ordinary particles (like electron), and their effect is manifested as gravitational interaction.

Loops intersect with each other to form a network, which is called spin network. When this network is observed over time, it is called spin foam. There are two values in this network which are important; nodes and links. They are related to elementary values of volume and area respectively Figure 1(b). A node stands for an elementary quantum (chunk) of volume, and similarly, a link stands for an elementary quantum of area.There are minimum values for volume and area that can exist within the framework of this theory. By a rough approximation, they are about one cubic Planck length, and one square Planck length respectively. This constitutes the granular aspect of the space or simply, the spatial granules. But despite of its precise description for the geometry of the spacetime at the Planck length scale, LQG is just a hypothetical approach that has not been yet validated by any experiment or observation. However, in this paper, we

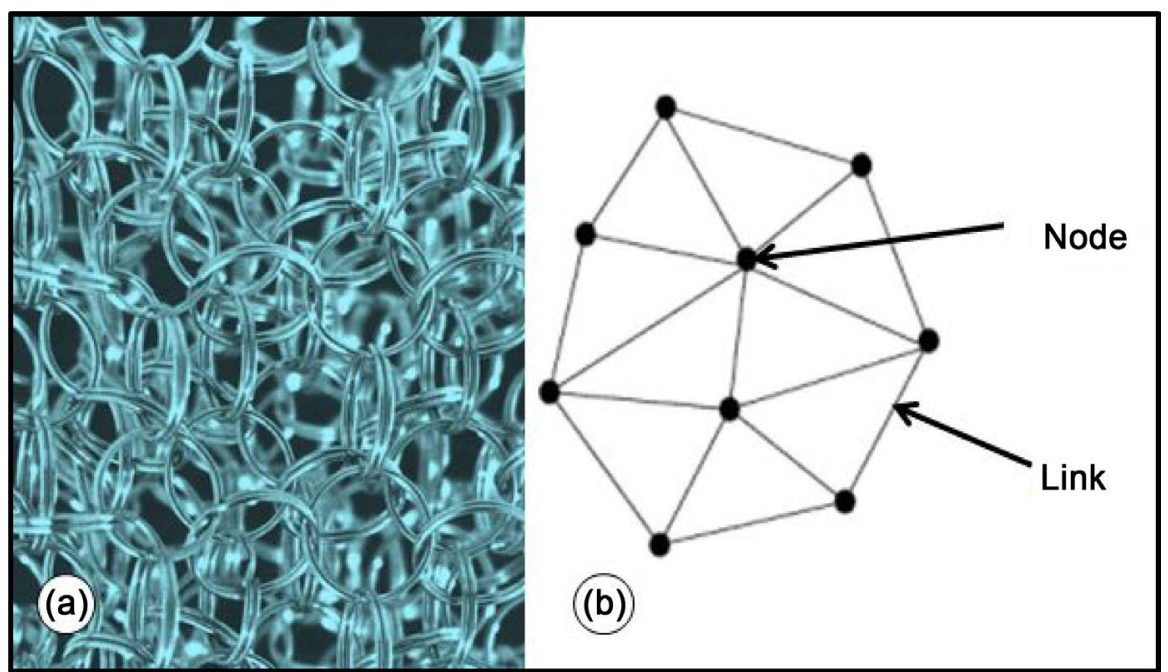

Figure 1. (a) Loops of LQG, they do not rely on space, they are the space; (b) Loops intersect with each other to form a network. This network is described by nodes and links. 
shall discuss two missed (unnoted) observations that prove the existence of discrete structure for the spacetime. But they cannot validate the precise shape of the quantized geometry which is predicted by LQG.

\section{Defining a Simple Approach to Detect Discreteness}

From our daily experience, we are familiar with two types of quantities; discrete and continuous quantities. To explain them, let us take the following example. Consider two types of bags. The first is a usual bag that is used to carry weight. The second one is a ball bag (mesh sack) which is used to carry soccer balls. The maximum capacity of the first bag is 10 kilograms, and for the second are 10 soccer balls Figure 2. Regarding the first bag which carries weight, we are familiar with the fact that matter is composed of discrete entities; molecular and sub-molecular particles. But this discreteness does not appear in our ordinary macroscopic measurements, as in this example. Therefore, just for the sake of demonstration, we shall consider it a continuous quantity. Later on, we shall discuss two accurate examples.

Now, let us guess the amount (quantity) that each bag carries without looking at them. Our possible answer regarding the first bag is any value from zero (empty bag) to $10 \mathrm{kgs}$ (full capacity). All the possible values from zero to ten kilograms are expected, which include values like $0.3 \mathrm{~kg}, 2.5 \mathrm{kgs}$, and $7.9 \mathrm{kgs}$. Concerning the second bag, our answer will be different. There are only eleven possibilities. Our possible answer will be any value in the range from zero (empty bag), one ball, 2 balls, 3 balls... up to 10 balls (maximum capacity). Only 11 answers are allowed. Values like 2.5 balls or 7.4 balls are not possible, because there are no 0.5 ball or 0.4 ball.

We shall use the term "spectrum" which refers to a set (or a continuum) of the possible values for each bag, and here you can simply notice the difference between the two spectrums of these bags. The spectrum of the second bag (ball bag)

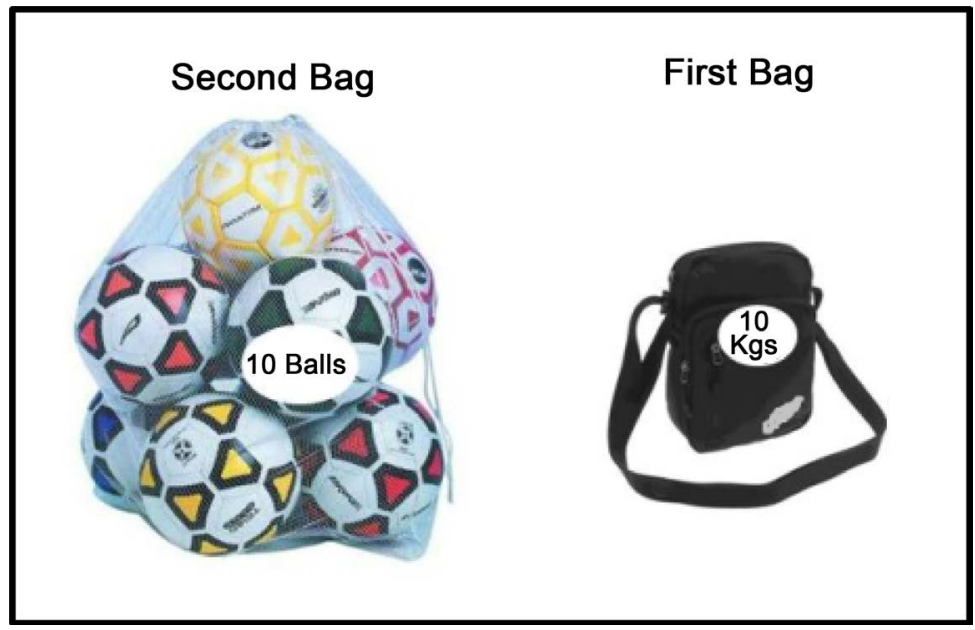

Figure 2. Two bags, the first one carries weight, which is considered from our daily macroscopic experience as a continuous quantity. The second bag, carries balls, which are discrete entities. 


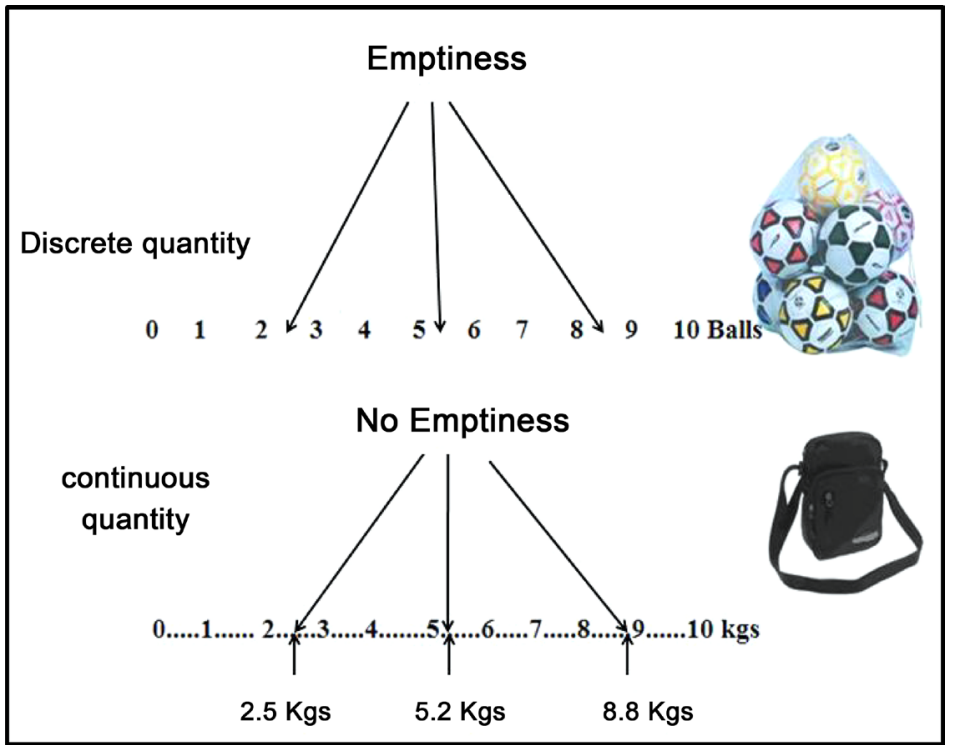

Figure 3. Discrete quantity versus continuous quantity, the difference between them is the existence of emptiness within the spectrum of a discrete quantity.

has sites of emptiness between its values Figure 3. There is nothing exists between any two successive values. For example 5 balls and 6 balls are two successive values within the spectrum. No possible value exists between them, because values like 5.7 or 5.3 balls do not exist; nothing are called 5.7 balls or 5.3 balls. Balls are discrete entities, and this discreteness is directly reflected as emptiness within the spectrum between the successive values Figure 3. This emptiness exists because the number of balls which is carried by this bag is a discrete quantity. On the other hand, the spectrum of the first bag is continuous, and there is no emptiness between the successive values Figure 3. There is always a possible value between any two successive values. From here, we consider it as a continuous quantity.

Let us take another example, which is the one dimensional quantum harmonic oscillator. The energy of this oscillator is a discrete quantity, and it is given by the equation $\left(n+\frac{1}{2}\right) h v$, where $(n=0,1,2,3, \cdots) v$ is the oscillator's frequency and $h$ is Planck's constant. In this section, we define the spectrum for a physical quantity as a set (or a continuum) of the possible values that this quantity can take or obtain. From this definition, and by using the previous equation, we can write part of the spectrum for the oscillator's energy as: $\frac{1}{2} h v, 1 \frac{1}{2} h v, 2 \frac{1}{2} h v, 3 \frac{1}{2} h v, 4 \frac{1}{2} h v, \cdots$. As you can see, the discreteness or the discontinuity of this physical quantity is directly reflected as emptiness within its spectrum. To understand what we mean by emptiness, let us ask ourselves a simple question; what is the number of possible values between $\frac{1}{2} h v$ and $1 \frac{1}{2} h v$ for this physical quantity? By looking at the spectrum, our answer will be zero. Nothing exists between $\frac{1}{2} h v$ and 
$1 \frac{1}{2} h v$ in the spectrum, because values like $\frac{2}{3} h v$ or $1 \frac{1}{4} h v$ do not exist. In other words, there is a complete absence of any possible value in the spectrum between the two successive values. From here, we call it emptiness in the spectrum between $\frac{1}{2} h v$ and $1 \frac{1}{2} h v$. As you can see in the spectrum, emptiness exists between any two successive values, and its existence is a direct consequence for the discreteness of this physical quantity Figure 4. On the other hand, a continuous quantity has a continuous spectrum which lacks the emptiness within it. Take for the example the spectrum of a classical kinetic energy for a moving particle. By using the equation $\frac{1}{2} m v^{2}$, we can write part of its spectrum as: $\frac{1}{2} m v_{\alpha}^{2}, \cdots, \frac{1}{2} m v_{\beta}^{2}, \cdots, \frac{1}{2} m v_{\gamma}^{2}, \cdots$ where $\frac{1}{2} m v_{\alpha}^{2}, \frac{1}{2} m v_{\beta}^{2}$ and $\frac{1}{2} m v_{\gamma}^{2}$ are three successive values within the spectrum. As you can see, the spectrum lacks the emptiness within it. Between any two successive values, there is always a possible, since $(v)$ is classically considered as a continuous quantity. Therefore, the number of possible values between the two successive values $\frac{1}{2} m v_{\alpha}^{2}$ and $\frac{1}{2} m v_{\beta}^{2}$ in the spectrum is infinite. Hence, the spectrum is continuous everywhere between the three successive values, and at any site between them there is a possible value. No emptiness exists, and from here, we call it a continuous quantity Figure 4.

Our final example is the electric charge. It is a discrete quantity. For a positively charged particle, its spectrum is $e, 2 e, 3 e, 4 e, 5 e, \cdots$. where " $e$ " is the elementary charge $\left(\approx 1.6 \times 10^{-19}\right.$ Coulombs). The sub-nucleic particles (Quarks) have smaller charges, but they are only found in combination. Now, in the spectrum above, you can notice that the number of possible values between any two

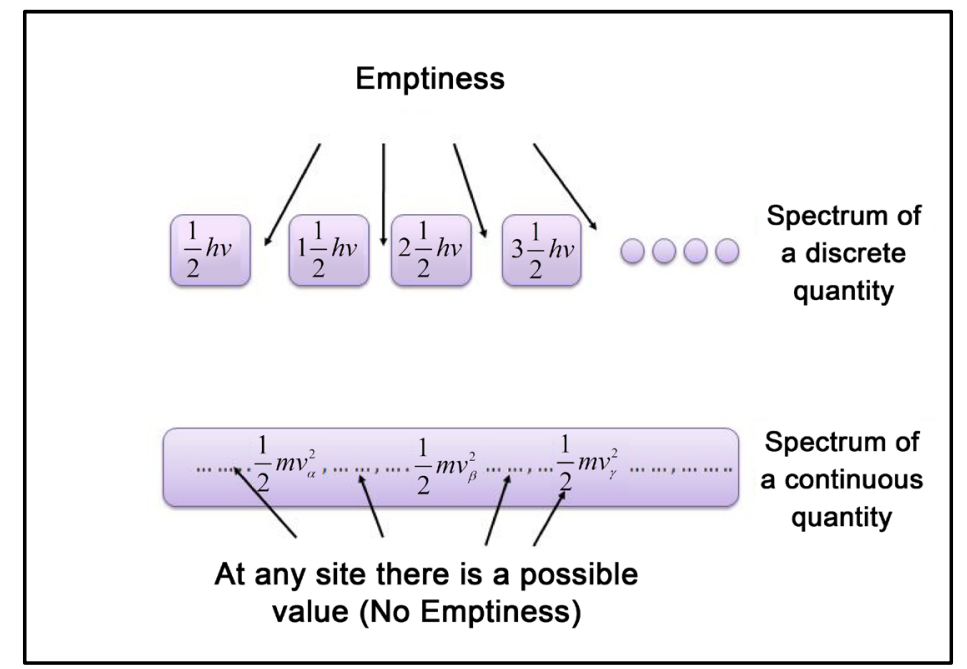

Figure 4. Regarding the quantum harmonic oscillator, as you can see, nothing exists between the successive values within the spectrum; there is a complete absence of any possible value between them. We call it emptiness within the spectrum. On the other hand, the spectrum of a continuous quantity lacks the emptiness within it; each small dot refers to a possible value within the spectrum. 
successive values is zero. For example, between $2 e$ and $3 e$, values like $2 \frac{1}{4} e$ or $2 \frac{3}{4} e$ do not exist. From here, there is emptiness in the spectrum between $2 e$ and $3 e$. The emptiness stands for the complete absence of any possible value within the spectrum between $2 e$ and $3 e$. Its existence is a direct reflection (consequence) for the discreteness of this physical quantity.

We can consider further examples for discrete quantities, but our conclusion will always be the same; a discrete physical quantity has a discontinuous spectrum of possible values. The discontinuity appears as emptiness between the spectrum's successive values. Emptiness by itself stands for the complete absence of any possible value within the spectrum. You can notice this in Figure 3 and Figure 4. On the other hand, a continuous quantity has a continuous spectrum. Emptiness does not exist, and the spectrum is continuous everywhere. This differentiates a discrete quantity from a continuous quantity, and from here, we can simply detect the discreteness of a physical quantity by observing the emptiness that exists within its spectrum.

\section{Time as a Physical Quantity, Does Its Spectrum Contain Emptiness?}

The nature of time is a site of controversy and ambiguity. But from an objective aspect, in physics, it is usually defined by its measurements; time is a quantity that is measured by a clock. In the universe, both space and time are merged together into one single entity called spacetime $(x, y, z, t)$, which is an inevitable consequence, regarding the theory of relativity Initially, in order to obtain a spectrum for time, we shall consider an observer within a specific frame of reference. In this frame of reference, time measurements are differences within time $(\Delta t)$, and these differences are measured or taken at the same spatial location within the frame of reference. Therefore, there should be no difference in the spatial coordinates $(\Delta x=\Delta y=\Delta z=0)$, but only difference in time. From here, regarding time measurement, we shall consider one axis; time axis $(c t)$ Figure 5.

As you can see from the figure, we consider our observer at specific moment $\left(t_{A}\right)$. Then, he (she) will have three components of time; the past, the present, and the future. The present is the time that exists between the future, and the past; it is the moment of now. The past is the period of time before the present. The future is the time that will come after the present. From a simple approach, the future can be visualized as a continuum of futuristic moments. A futuristic moment is defined as the moment of the present after a flow of a specific time interval. For example, from Figure $5,\left(t_{B}\right)$ and $\left(t_{C}\right)$ are two futuristic moments that will be the present for our observer after a flow of $\left(t_{B}-t_{A}\right)$, and $\left(t_{C}-t_{A}\right)$ respectively.

In the previous section, we have defined the spectrum for a physical quantity as a set (or continuum) of the possible values that this quantity can take. For our 


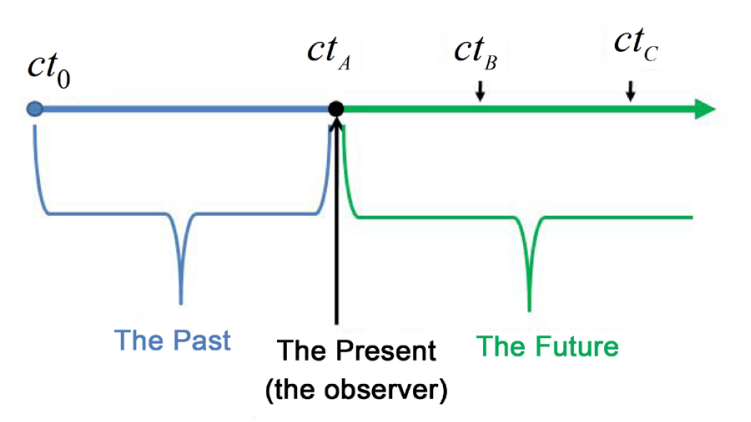

Figure 5. Time axis; the past, the present, and the future for our observer. We consider our observer at the moment $t_{A}$ (the present for him), $t_{0}$ is defined as the moment at which observations have been started regarding this frame of reference.

observer, and by looking at the axis, the timing of any event $(t)$ can take any possible value from the past $t<t_{A}$ (the event occurred), the present $t=t_{A}$ (the event is occurring now), up to the future $t>t_{A}$ (the event will occur later). Therefore, all the possible time values that exist in the time axis Figure 5 are included within the spectrum of time. From here, the spectrum of time regarding our observer is composed of all the possible values that appear in time axis; the past, the future, and the present. In other words, it can be regarded as the axis itself.

Now, I think we can ask ourselves a simple question; time, does it flow continuously like a smooth river? The fundamental difference between science and philosophy is that science is composed of concepts that are validated by practical evidences, where philosophy is not. Practical evidences are experiments, and observations. Both are subjected to recording and measurements. Regarding observations, by looking at the previous figure. I believe this is one of the simplest observations in physics that can be used to validate a proposed hypothesis (discreteness of time). It is one of the simplest because you do not have to measure anything to observe the emptiness that exists between the future and the present. As we have discussed in the previous section, emptiness stands for the complete absence of any possible value within the spectrum, and as you can see in the spectrum of time (time axis), the total number of possible values (time values) that exist between the present $\left(t_{A}\right)$ and the future is zero; there is a complete absence of any possible value in the spectrum between the future and the present Figure 6.

The future is a continuum of futuristic moments. From here, the emptiness that exists between the present and the future is just an emptiness between the present and a futuristic moment; call it $\left(t_{\alpha}\right)$. Regarding, our observer, when time flows by the amount $\left(t_{\alpha}-t_{A}\right)$. Then, $\left(t_{\alpha}\right)$ will not be a futuristic moment any more. It is now the present $\left(t=t_{\alpha}\right)$, and similarly as the previous $\left(t_{A}\right)$, there will be emptiness between it, and the future. Again, the future is just a continuum of futuristic moments. Therefore, the emptiness that exists between the present, $\left(t_{\alpha}\right)$ and the future is just emptiness between the present and a 


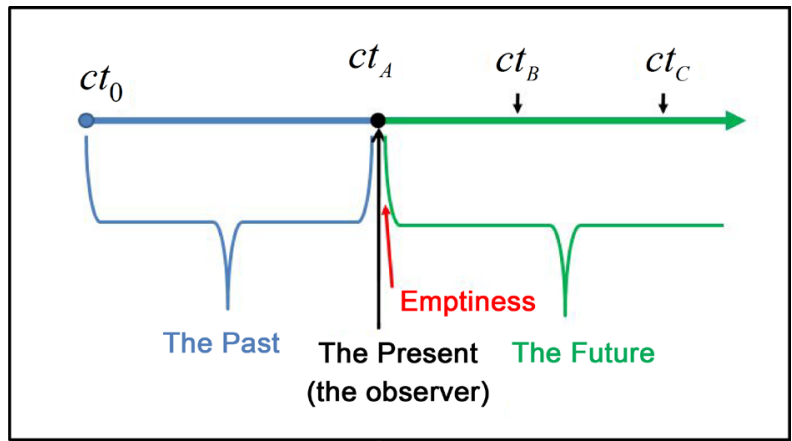

Figure 6. As you can see in the time spectrum (axis), nothing exists between the present (represented by the black dot) and the future (represented by the straight green line); there is a complete absence of any possible value between them. Emptiness also exists between the present and the past. But this has no physical significance or meaning. Because time as we currently know advances to the future, and it does not flow reversely to the past.

futuristic moment, call it $\left(t_{\alpha 2}\right)$. By repeating the previous analysis, we can get $\left(t_{\alpha 3}\right),\left(t_{\alpha 4}\right)$ and so on. From here, we can see that time flows by a certain pattern; $t_{A}, t_{\alpha}, t_{\alpha 2}, t_{\alpha 3}, t_{\alpha 4}, t_{\alpha 5}, t_{\alpha 6}, \cdots$ which is discrete, and not continuous.

Time does not flow continuously like a smooth river due to a simple observation which is absence of any possible value of time that can exist between the present, and the future. It flows in certain jumps like a ticking of a clock. Theoretically, the magnitude of $\left(t_{\alpha}-t_{A}\right)$ or (the quantum of time) is very small. It is expected to be approximately about the value of Planck time $\left(\sqrt{\hbar G / c^{5}} \approx 10^{-43} \mathrm{~s}\right)$. From here, one minute is divided to 60 seconds, and approximately, each second is divided to $10^{43}$ elementary time quanta, therefore, time discreteness-as we currently observe-does not play any apparent role in our ordinary macroscopic activities. Its significance becomes obvious at the scale, where the theory of quantum gravity works. At that length scale, as we have discussed earlier, LQG visualizes the space by a spin network, which describes the quantized microscopic geometry of the space by using nodes and links. This spin network, when it is observed over time, its name changes to a spin foam. An important point that should be mentioned is that the geometry of this microscopic space is not fixed. But it changes with time for a number of reasons which include matter movement and the quantum effects of the uncertainty principle (quantum fluctuations). These geometrical changes appear as rearrangements within the patterns that nodes and links can take within the spin network (e.g. multiple nodes may combine to form a single node). At the level of these events, the discreteness of time becomes important. Because it implies that these rearrangements which happen within the spin network, will not occur in a smooth, continuous pattern, because time does not flow continuously. Instead of this, it will occur in discrete abrupt steps, since time advances in a discrete pattern. From this perspective, and at this small level, time can be defined by the sequence of distinct moves that rearrange the network. More precisely, I quote the following words from the American physicist Lee Smolin, one of the theorists who developed the LQG 
"Time in our universe flows by the ticking of innumerable clocks-in a sense, at every location in the spin foam where a quantum "move" takes place, a clock at that location has ticked once" [3].

\section{Position as a Physical Quantity, Does Its Spectrum Contain Emptiness?}

It is logical to assume that time discreteness should be associated with space discreteness, since space and time are intimately connected in nature, and they constitute one physical entity (spacetime), where time is a dimension within its structure. However, spatial measurements within the spacetime are concerned with the differences between the spatial coordinates. These differences are measured or taken at the same moment, which means there is no difference in time $(\Delta t=0)$, but only differences in $(\Delta x, \Delta y, \Delta z)$ which are the spatial component of the spacetime. Now, if we consider position as a physical quantity, we shall ask ourselves an important question. Is it continuous or discrete? To answer this question, we start with one spatial dimension (a line) for simplicity. Positions in this line are represented with axis $(x)$. The position spectrum represents all the possible values that a position can take. It can take a positive or a negative value (to the right or to the left with respect to the origin), or it can be the origin itself. It is illustrated on the axis by labeling the axis with position units, as shown in Figure 7 below.

By looking at the spectrum above, there are three facts that are noticeable; the First fact from Figure 7, the positive part of the spectrum represents a continuum of positions that are directed in the positive direction with respect to the origin. The negative part of the spectrum represents a continuum of positions that are directed in the opposite direction with respect to the origin. Therefore, the word "positive" or "negative" only refers to the direction. Positive means to the right and negative means to the left with respect to the origin. The second fact; existence of the origin position in the spectrum which is the position that is located outside the positive and the negative parts of the spectrum. Therefore, it is neutral (null vector). The final fact; in the spectrum above, the number of possible values (positions) between the positive part and the negative part of the spectrum is one, which is the origin itself, but the number of possible values (positions) between the positive part of the spectrum and the origin is zero.

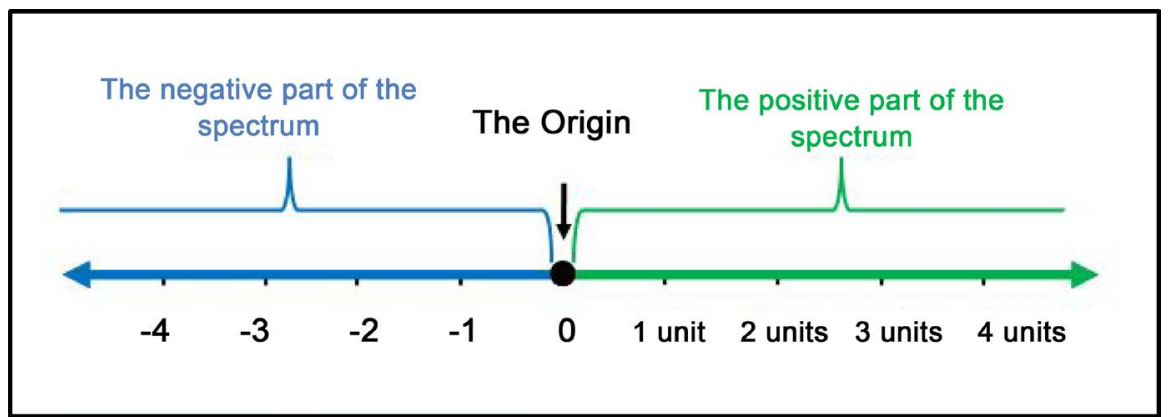

Figure 7. A line with Position Spectrum. 
Regarding observations in physics, by looking at the previous figure. I believe this is one of the simplest observations in physics that can be used to validate a proposed hypothesis (spatial discreteness). It is one of the simplest, because you do not have to measure anything to observe the emptiness in the spectrum that exists between the origin and the positive (or the negative) part of the spectrum Figure 7. As you can see in Figure 7, between the origin and the positive part of the spectrum, the total number of possible values (positions) is zero; there is a complete absence of any possible value within the spectrum between the origin and the positive part. It is emptiness within the spectrum Figure 8.

Since the positive part of the spectrum is merely a continuum of positive positions. Then, the emptiness that exists between the origin and the positive part of the spectrum is an emptiness between the origin and a positive position, call it position $\left(x_{1}\right)$. The origin position is a relative position and not an absolute. Therefore, position $\left(x_{1}\right)$ can be considered as an origin position. Then there will be emptiness between $\left(x_{1}\right)$ and the following position in the spectrum, just like the previous one, call it position $\left(x_{2}\right)$. Also position $\left(x_{2}\right)$ can be considered as an origin, since the origin is a relative concept. Therefore, there will be emptiness between $\left(x_{2}\right)$ and the following position in the spectrum, call this position $\left(x_{3}\right)$, and so on. Therefore, the position spectrum in the positive direction will take the form: $0, x_{1}, x_{2}, x_{3}, x_{4} \ldots$ which is discrete and not continuous, and the number of possible positions in axis interval $(\Delta x)$ is limited, and not infinite, but what does it mean?

Initially it means that position is a discrete physical quantity. It is important to note that our analysis above can only prove the discreteness of the position as a physical value. But it cannot answer whether the successive positions are equally spaced or not, but it only shows that they are spaced. Classical geometry defines line as a continuum of infinite number of points spreading in one dimension. This definition makes any given value of length $(\Delta x)$ infinitely divisible Figure 9.

In this classical definition, any point in the line refers to a position in space. Therefore, existence of infinite number points in any given value of length $(\Delta x)$ means existence of infinite number of positions in that length. This clearly

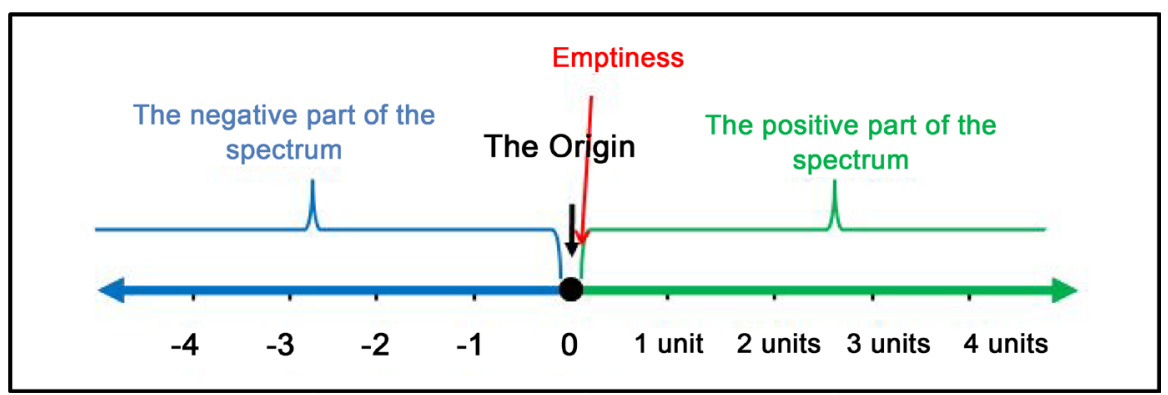

Figure 8. As you can see in the spectrum (axis), nothing exists between the origin (represented by the black dot) and the positive part of the spectrum (represented by the straight green line). There is a complete absence of any possible value between them; it is emptiness within the spectrum. Also, emptiness exists between the origin and the negative part of the spectrum. 


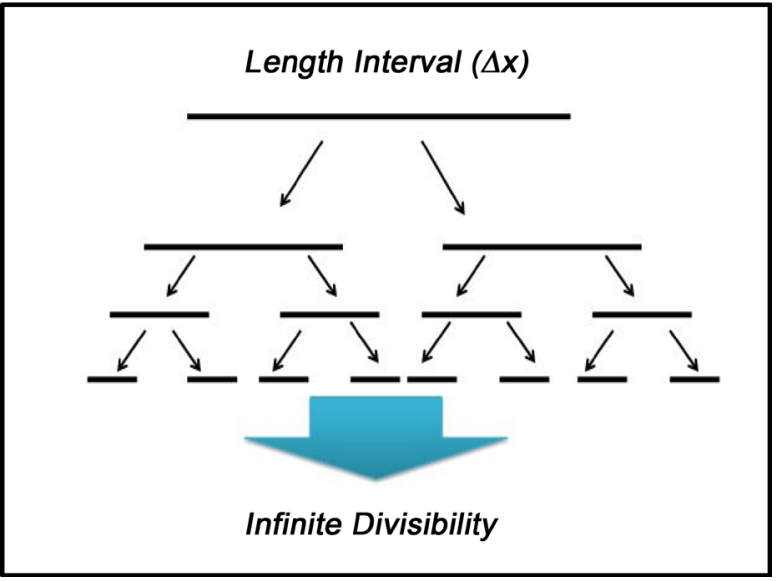

Figure 9. Line as a continuum of points. It is infinitely divisible. Infinite number of positions in the length interval $(\Delta \mathrm{x})$ results from the infinite number of points.

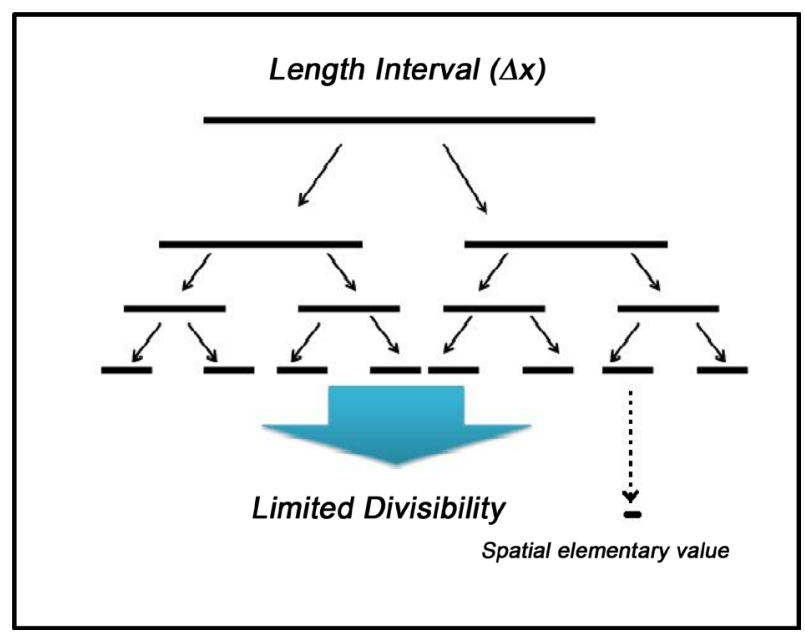

Figure 10. Line as a continuum of spatial quanta results in limited divisibility. Limited number of positions results from limited number of quanta in the interval $(\Delta x)$.

contradicts the discreteness of position as a physical value. Because discreteness implies that the number of positions in any length value is limited, as we have shown in the analysis above. From here, a new definition of line is required to solve this contradiction. By redefining line as a continuum of quanta instead of points, the problem is simply solved. Each quantum will represent-or refer to-a position in space and since the quantum has a non-zero value of length, their number in any length interval $(\Delta x)$ is limited. This in turn results in existence of limited number of positions in that interval, which is consistent with its discreteness concept Figure 10.

The quantum means an elementary value of length. The word "elementary" means it is not divisible, just like elementary particles are not divisible. Therefore, observation of space below the level of the quantum is not possible, because it results in the divisibility of the quantum itself, and this cannot happen, since it is elementary.

Existence of elementary value for length implies the existence of elementary 
values for area and volume too. We shall consider the following example. From the previous discussion, existence of the origin position illustrates discreteness in space structure, but it does not illustrate a specific or certain shape of microscopic geometry at the discreteness length scale. This creates a problem when trying to extend the previous conclusion about space discreteness to include two and three spatial dimensions. However, the problem is solved by using a large length scale relative to the scale of discreteness. Because at this large scale, the microscopic discrete geometry is reduced to the classical macroscopic geometry as an approximation (just as the classical mechanics is used as an approximation for quantum mechanics at the macroscopic length scale).

Therefore by choosing a large macroscopic length scale, the classical Cartesian coordinates system is used as an approximation, but it is important to bear in mind that the axes $(x),(y)$ and $(z)$ are discrete and not continuous, since they contain an origin position. By considering areas, an additional spatial dimension $(y)$ is required, and it is discrete just like $(x)$, since it contains an origin position. The "classical" definition of area is that it represents a two dimensional continuum of infinite number of points, and this definition makes any given value of area infinitely divisible. This definition contradicts the conclusion regarding position discreteness as will be illustrated below. Let us take a circle as an example, from the classical definition of area; it represents a continuum of infinite number of points spreading in two dimensions. Since every point in the circle refers to -or represents- a position in space, existence of infinite number of points in the circle result in existence of infinite number of positions inside it. This in turn results in existence of infinite number of positions in $(\Delta x)$ and $(\Delta y)$ intervals which bound this circle as illustrated in Figure 11. This clearly contradicts the fact of position discreteness.

This happens because every position in the circle refers to a position in $(x)$ and $(y)$ axes, for example, position or point number (1) in the circle refers to position $\left(x_{1}, y_{1}\right)$ in the axes. Position or point number (2) in the circle refers to position $\left(x_{2}, y_{2}\right)$, and point or position number (3) will refer to position $\left(x_{3}, y_{3}\right)$ in the circle and so on.

Since the number of points inside the circle is infinite, this results in infinite

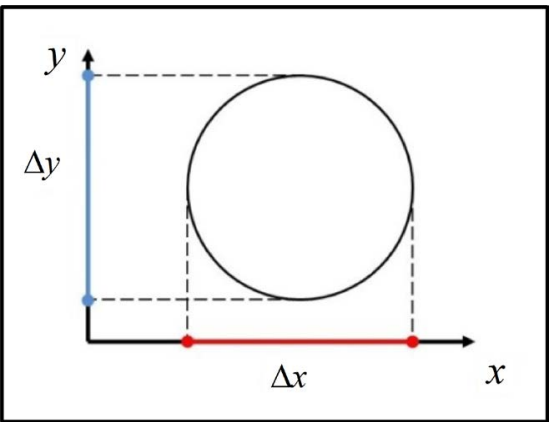

Figure 11. By taking a large scale, classical geometry is used as an approximation for the quantized, microscopic geometry. Just as the classical mechanics is used as an approximation for the quantum mechanics at the macroscopic length scale. 
number of positions in $(\Delta x)$ and $(\Delta y)$ intervals, as illustrated in Figure 12 below.

The previous result makes a contradiction with the result regarding position discreteness, because the number of positions in $(\Delta x)$ and $(\Delta y)$ intervals will be infinite and not limited as we have shown before. By redefining area as a continuum of quanta, instead of points, the spatial quantum represents an elementary value of area (not divisible).

The quantum of area is an elementary value. In physics elementary values are not divisible, just like elementary particles. From here, the quantum cannot refer to more than one position in space. Because observation of the space below the quantum's level is not possible. It will result in the divisibility of the quantum itself, and this cannot happen, since it is an elementary value. Therefore, every quantum will refer to a single position inside the circle. Since the quantum has a non-zero value of area, the number of quanta, and therefore positions inside the circle will be limited. Now, since the Cartesian coordinates are used as an approximation, every position in the circle is approximated to a position in $(x)$ and $(y)$ axes. Position number (1) in the circle is approximated to position $\left(x_{1}, y_{1}\right)$ in the axes, position number (2) in the circle is approximated to position $\left(x_{2}, y_{2}\right)$ and so on, just like the idea from Figure 12. The number of positions inside the circle will be limited. This results in a limited number of positions in $(\Delta x)$ and $(\Delta y)$ intervals which bound the circle's area. This is consistent with the fact of position discreteness. By considering volumes, the same concept used in dealing with area is hold here, but with additional dimension $(z)$ because volume is a three dimensional quantity. This leads to redefining volumes as a continuum of three dimensional quanta, instead of points.

Existence of elementary values for space, which we can call it spatial granules may appear strange, but let us recall a historical similarity. Before the $19^{\text {th }}$ century, many scientists believed that matter is infinitely divisible, which means that it is not composed of elementary constituents The idea of elementary constituents (elementary particles) of matter was a strange too. But today, we know that matter is composed of atomic and subatomic particles. Space has the same

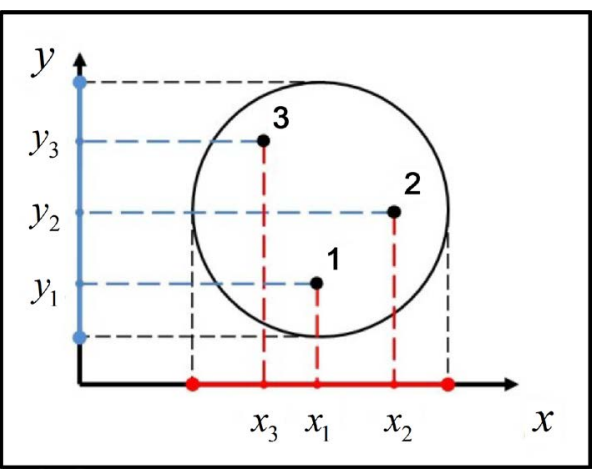

Figure 12. Existence of infinite number of positions inside the circle, results in infinite number of positions in $(\Delta x)$ and $(\Delta y)$ intervals. Since every position in the circle refers to a position in the intervals. 
argument. Therefore, in science, we have to follow the evidence, regardless of its impression. On the other hand, the existence of the spatial granules solves one of the major problems in physics, which is the infinities problem. This problem was originated in 1927, when the German physicist Werner Heisenberg had discovered a new principle, which limits our knowledge in certain measurements. It is called the uncertainty principle. By considering the uncertainty principle, infinities in calculations arise from physical interactions that occur in spatial points, which lead to results that contradict our practical observations. But now, this problem disappears very simply. Because we know that these infinities do not exist because there are no spatial points. The space has elementary spatial granules that restrict the uncertainty principle, and preventing it from blowing up.

Generally speaking, and apart from the picture that is given by LQG for the granular space (links and nodes). Regarding space discreteness as an idea, once I was asked a question; if the space has a discontinuous structure which means that it consists of elementary quanta or granules. This discontinuity indicates that the space will end at the limits of each spatial quantum or granule. This in turn results in existence of gaps or a space-less physical entity that will separate these granules, just like the elementary constituents of matter (particles) are separated by space. These gaps should not have any space; since they separate the space itself (separate the spatial granules). From here, they are space-less physical entities Figure 13. Then, how can we move from one place to another through the space? Or, how can physical entities like waves move or pass through these physical gaps that contains no space (space-less entity), when they propagate from one location to another ? Because movement as a physical concept is concerned with space. It is the change of position with time, and position exists within the space, but not outside the space. Therefore, we can only move through the space (spatial granules), but we cannot move outside the space (space-less entities).

I think this question can be answered from a simple perspective [9]. The value of space (length, width, and height) in the space-less physical entity (gaps) is

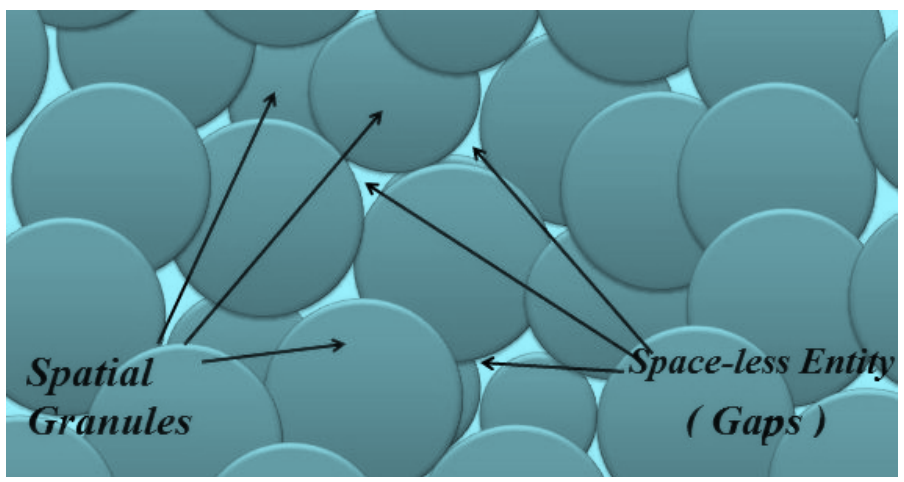

Figure 13. Imaginary description of the granular space. The space-less physical entity which separates the spatial granules is dimensionless; it has no length, height or width. It is represented in this figure as gaps (or spaces) between the spatial granules just for the sake of demonstration. 
zero, since it has no space. Therefore, it is a dimensionless entity. We know from our classical geometry that dimensionless entities are spatial points. Because in geometry (and also physics), points do not have length, width or height. Therefore, they are dimensionless. From here, spatial points and the space-less entity are physically indistinguishable. Both share the same concept. Therefore, the statement that says "the spatial granules are separated by gaps or space-less entity" is physically equivalent to the description that implies" the spatial granules are separated by virtual spatial points". We call it "virtual", because they do not play any role with the physical interactions. Because as we know, physical interactions occur within space, which means within the spatial granules. Bu they do not occur outside the space (the space-less entity), which appears to us as spatial points. Therefore, their only apparent role is to allow a linkage or connection between the space granules, which in turn allow us to move through a discrete space without any problem.

This also may explain why the space appears so smooth and continuous on the large scale, although it has a discontinuous structure. For the sake of clarification, a volume of frozen water (ice) appears so smooth and continuous at the large macroscopic level, although microscopically, it consists of discrete, discontinuous molecules. The reason behind this is that these separate, discontinuous molecules are linked to each other through the intermolecular forces, which hold these molecules as one continuous unity at the large level. By a "rough" resemblance, the space can be visualized from a similar perspective. Microscopically, it consists of discrete, discontinuous granules. These granules are linked or connected to each other through virtual spatial points (space-less physical entity). But at the large macroscopic level, the value of one spatial granule (volume and area) becomes extremely small to be notable. Therefore, it can be reduced to a spatial point as sort of approximation; an approximate point. These approximate points (spatial granules) will not be discrete or discontinuous, because they will be linked to each other through the virtual spatial points. From here, at the large scale, the discrete space can be visualized as a continuum of spatial points (approximate and virtual), just like the classical continuous space is considered to be a continuum of spatial points.

\section{Discussion and Conclusion}

I believe in this paper, we have proven the existence of a discrete structure for the spacetime by a very simple approach. Time flows in discrete steps, and space is not infinitely divisible. Therefore, the major prediction of LQG is validated. This represents a successful aspect for this theory, and shows that LQG is on the right direction, because it yields spacetime discreteness as a prediction from its theoretical framework, and does not use it as an assumption or a postulate. But our problem is that our evidences do not validate the (physical) geometrical features for the discrete spacetime which is given by LQG. Therefore, the following question is problematic; do the spatial granules have the same geometrical fea- 
tures that are provided to us by the LQG? The possibility that one day, another theory may emerge to the surface, and contains a discrete picture for the space, but with geometrical features that are entirely different from that of LQG. At that moment, unless we have an experiment or observation that can tell us the accurate description of this microscopic, quantized geometry. Then, the validity of each one of them will be questionable. I hope we can find an answer soon. But for now, I am excited, because we know that the spatial granules do exist, although we do not know their geometrical (physical) features. Their existence alone can explain how the uncertainty principle and the general theory of relativity exist in our universe without their usual conflict. Certainly, this is promising.

\section{Acknowledgements}

The publication of this article was funded by the quantum gravity group of the centre de physique théorique de Luminy, France.

\section{References}

[1] Rovelli, C. (2004) Quantum Gravity. Cambridge University Press, Cambridge. https://doi.org/10.1017/CBO9780511755804

[2] Gambini, R. and Pullin, J. (2011) A First Course in Loop Quantum Gravity. Oxford University Press, Oxford. https://doi.org/10.1093/acprof:oso/9780199590759.001.0001

[3] Smolin, L. (2004) Scientific American, 290, 66-75. https://doi.org/10.1038/scientificamerican0104-66

[4] Davies, P.C.W. and Brown, J., Eds. (1988) Superstrings: A Theory of Everything? Cambridge University Press, Cambridge.

[5] Barrau, A., Cailleteau, T., Cao, X., Diaz-Polo, J. and Grain, J. (2011) Physical Review Letters, 107, 251-301. https://doi.org/10.1103/PhysRevLett.107.251301

[6] Hawking, S.W. (1974) Nature, 248, 30-31. https://doi.org/10.1038/248030a0

[7] Einstein, A. (1961) Relativity: The Special and the General Theory. Three Rivers Press, New York.

[8] Carroll, S. (2004) Spacetime and Geometry: An Introduction to General Relativity. Addison-Wesley.

[9] Isam, A. (2016) The Perfect Universe: A New Perspective for Light, Matter, and Gravity. Noor Publishing. 